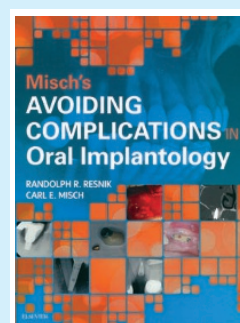

\section{MISCH'S AVOIDING COMPLICATIONS IN ORAL IMPLANTOLOGY}

Carl E. Misch and Randolph Resnik

Elsevier; 2017

price $f 150$ pp. 912

ISBN: 9780323375801

This comprehensive text is a guide to the diagnosis, aetiology and management of complications in oral implantology. It is co-written by one of the giants of modern dental implantology, Carl Misch. It is extensive in detail and covers almost every possible complication that may occur in oral implants. It even covers unusual occurrences such as management of a fire in the surgery. The intended audience is clinicians with some clinical experience of dental implants, though there is much detail on the principles of implants. The book has five parts covering complications in diagnosis and treatment planning, surgical, prosthetic, periodontal and maintenance issues and medico-legal aspects. These parts are divided over 19 chapters.

As the title suggests, the book mainly deals with the prevention of complications rather than their management. In this regard it is very different to other implant complication texts such as Froum's dental implant complications. Therefore, there is a huge emphasis on delivering an evidence-based understanding of the principles of each area, to prevent a complication from ever occurring. Each chapter covers complications related to that area. The chapters are divided into different complications. At the start of each, the reader is provided with a solid foundation of core knowledge followed by the associated complication, aetiology, prevention and then management.

I think this is an excellent book. It isn't really a reference guide as it's quite difficult to find information due to the book's volume and layout. If you want a book for the surgery in case a complication occurs then this isn't it and Froum's text would be a better bet. It is tough to sit down and digest its wealth of information but if you do you will be rewarded. Misch was renowned as being an innovative teacher and he presents some excellent drawings and tables to explain his ideas. I like how the reader is provided with the core knowledge and the emphasis on prevention before the treatment and management of the various complications. The book also covers so many complications that aren't included in other texts. There is an excellent summary table of articles on the most common implant complication along with their findings within the first chapter. The book, however, is let down slightly by the quality of many of the photographs which can be quite grainy at times. Overall, I would highly recommend the text to anyone involved in dental implants, wishing to further their knowledge.

Duncan Weir 